\title{
[ 240 ]
}

XLIX. Differtation Litteraire fur une Colonie Egiptiene etablie à Athènes: Prefentée à l'illuftre Academie des Antiquaires de Londres. Par Fred. Samuel Schmidt de Berne.

Read February 18, 1762.

TE trouve un plaifir fenfible à rechercher l' origine des arts et des fciences, et à fuivre les progrès qu'ils ont fait fucceffivement dans les tems de la plus haute antiquité. Je les vois naiffans et comme au berceau en Egipte; je les confidere dès là portés en Grèce ou ils montent infenfiblement au plus haut degré de perfection.

$E_{N}$ Egipte j'admire une noble fimplicité dans les arts, jointe à une grande folidité. En Grèce, j'y remarque beaucoup d' art, de feu, et une heureufe hardieffe.

Dans les fciences, je vois le fombre, le miftérieux Egiptien couvrir fes dogmes d'un voile épais, repandre l'obfcurité et les tenèbres fur fa doctrine: d'un autre côté j'obferve le Grec, amateur des nouveautés, faifant tous les jours des changemens à fa théologie, ajoutant, retranchant à fa fantaifie, et embrouillant fi fort cette fcience, qu'elle fe trouve aujourd'hui remplie de toutes fortes de comtradietions et d'abfurdités.

MAIs à travers ces changemens, je reconois toujours less Dieux Egiptiens dans la Gréce. Malgré tout ce que les Grecs ont pû faire pour cacher l'origine de leur théologie, il eft aifé de lever le mafque, et de découvrir qu'ils ont reçu leur culte des Egiptiens. Il eft aifé auffi de démontrer, que c'eft à l'Egipte que la Grèce eft redevable des arts.

POUR fe convaincre de cette vérité, il n'y a qu'à lire l'excellent ouvrage de Mr. le Comte de Caylus, qui fait voir en tant d'endroits, 
On an Egyptian Colony eftablifhed at Athens.

droits, avec évidence, l'affinité des monumens Egiptiens avec ceux des anciens Grecs.

Transportes vous en Grèce, examinés la, comme elle étoit dans l'antiquité la plus reculée; vous y verréz celebrer les miftères d'Ifis et d'Ofiris, tout comme ils etoient en Egipte: vous verrés les Athéniens offrants, au lieu de boeufs, des gâteaux qui en avoient l'empreinte, de l'eau, au lieu du vin, conformément aux rits Egiptiens; vòus verréz ce même peuple fe fervant de monö̈es fur lesquelles font repréfentés les Sphynx, animaux emblematiques des Egiptiens. Vous ne verréz que des ftatües de bois, d'une attitude fimple, et le plus ordinairement terminées en gaine; en un mot, vous ne croiréz point que vous foiéz en Grèce, mais plutôt en Egipte.

CETTE affinité eft fi grande et fi manifefte, que perfone ne peut nier qu'on n'en puiffe inferer avec raifon, qu'il y a eu une comunication entre ces' deux peuples; foit qu'elle fe foit faite par les Thraces, anciens coloniftes Egiptiens, qui enfuite allerent s'étaoblir en Grece, come je l'ai prouvé dans une differtation fur ce fujet: foit qu'elle doive être atribuée aux Egiptiens eux mêmes, qui en diférens tems ont conduit des colonies en Grèce, comme font celles d'Argos et d'Athènes. C'eft de cette derniére colonie que je me propofe de traiter dans cette differtation.

JE n'ai point deffein d'entrer dans tout le détail, où cette matiére me paroit naturellement inviter; mais je me bornerai plûtôt à l'éxamen .des preuves les plus fenfibles et les plus fortes, qui établiffent cette colonie: telles que font, I. les témuignages des anciens auteurs: 2. les rois d'Athenes Egiptiens: 3. le culte de Minerve d'Athenes, le même que celui de Mitha ou Minerve Egiptienne honorée à Saïs.

I. Platon, ce grand philofophe, au quel perfone ne fauroit refufer croïance, affûre, que les Saïtes $[a]$ ont une prédilection

[a] Edition de Serranus, Tome III. pag. 21. cet auteur dit des Saites, $\mu \alpha^{\prime} \lambda \alpha \delta_{\alpha}$

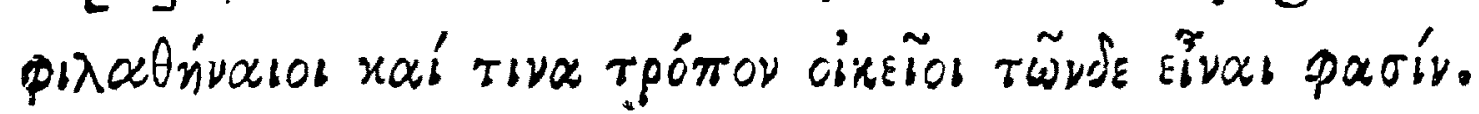

VoI..I.

$\mathrm{I} \mathrm{i}$

pous 
On an Egyntiun Colony efablifbed at Athens.

pour les Atiùniens, et qu'ils font en quelque façon leurs parents. Procle, dans fes commentaires fur le paffage du Timé [b], que je viens de citer, ajoute que les anciens font partagés à ce fujet; que Callifthènes et Phanodemus [c] avançoient que les Saïtes Egiptiens étoient une colonie des Athéniens; qu'au contraire Theopompus [d] foutenoit fans vanité, aïant uniquement égard au vrai, que les Athèniens étoient une colonie Egiptienne des Saites. Il avoit de fon côté le témoignage des Egiptiens, qui, entre plufieurs colonies forties de leur païs, fe vantoient à jufte titre d'en avoir conduit une à Athènes[e]. Diodore de Sicile nous l'aprend, en difant: " On affure que les Athèniens font une colonie des Saïtes, "c peuples de l'Egipte." Cet auteur s'étend fort au long là deffus, en alléguant toutes les raifons fur lefquelles ce fentiment eft fondé. Je les citerai à mefure que j'en aurai befoin, dans le cours de cette differtation, et je tâcherai de prouver, que Diodore a trop écouté l'ambition, et l'amour propre des Grecs, lors qu'en finiffant cet article il dit: "Voilà de quoi ceuxci fe vantent, avec "plus de zèle, à mon avis, pour la gloire de leur nation, que " pour la verité, en ajoutant, que la magnificence de leurs rois, " et le nombre prodigieux des premiers habitans de l'Egipte a " été la caufe des tranfmigrations que nous venons de marquer, et " de plufieurs autres, que nous paffons fous filence; parceque nous " ne les voïons foutenües d'aucune preuve affés fenfible, ou at" teftés par aucun monument affès certain."

II. LE conducteur de cette colonie de Saites étoit Cecrops; Jean Tfetzes l'affûre dans ces vers $[f]$ :

[b] Procle. liv. i, p. 30.

[c] Epitre d'Apoll. Thyaneus aux Saïtes.

[d] Eusèbe P. Evang. x. 10. p. 49 I.

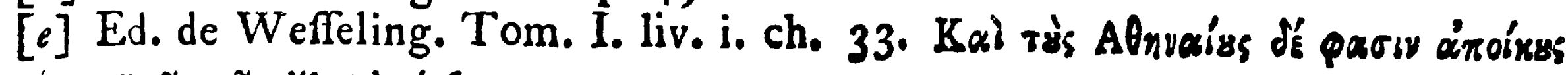

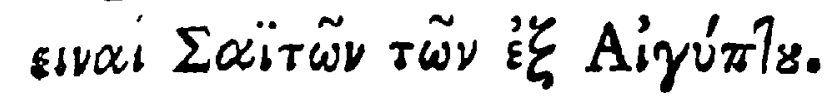

$[f]$ Var. Hift. Chil. xviii. ver. 28. 
On an Egyptian Colony eftablifked at Athens.

“ Út autem a Saï urbe Aegyptia,

"Poft Ogygium illud diluvium,

"Cecrops venit Athenas Graeciae [g].

SANs m'arrêter à un fait auffi conu, je tâcherai de prouver fa vérité par la langue Cofte, en démontrant, que le nom de Cecrops eft Egiptien, ce qui eft le plus fûr argument. pour prouver que l'Egipte eft le païs, d'où ce héros vint s'ètablir à Athènes.

CECRoPs étoit prononcé en Egipte Cecrop, car il eft certain, que c'étoit la coûtume des Grecs d'ajouter la lettre $s$ à la fin d'un mot finiffant par $p$; ainfi au lieu de Pelop, les Grecs difoient Pelops, et dans un autre cas Pelopa, preuve que la lettre $s$ n'eft pas de l'effence du mot. Cecrop, en Egiptien Gizpsq Sigraph, fignifie conjunctio, miftio, complexio [b]. C'eft éfectivement le nom que les anciens donnoient à Cecrops, qui eft apellé $\Delta$ ıquìs, ceft à dire " duplicis et mixtae naturae," et il exprime au jufte le mot de Sigraph Egiptien. Les auteurs, qui nous apprennent que Cecrops a été appelle de ce nom, font en grand nombre; mais ils difèrent beaucoup quant à l'explication qu'ils en donent. Il y en a qui croient qu'il porte ce nom, parce qu'il a eu en haut la forme d'un homme, et en bas celle d'un ferpent. Ariftophane [i] paroit favorifer cette expofition en falutant Cecrops de'cette maniere:

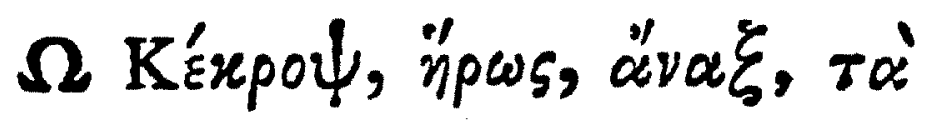

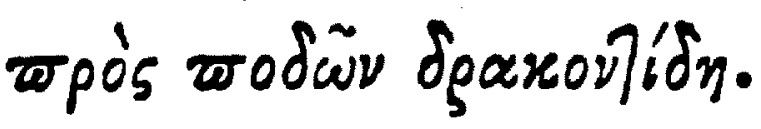

D'AUTRES affurrent, que c'eft parce qu'il fut le premier qui inttitua à Athènes le mariage. "Ante Deucalionis tempora," dit Juftin $[k]$, " Athenae regem habuere Cecropem, quem, ut omnis

[g] V. auffi Suidas, Cedrenus, et principalement Meurfius de Regg. Athenienf. liv.-i. ch. 6.

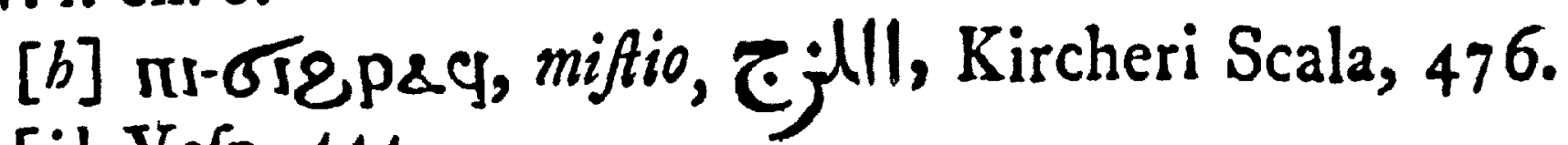

[i] Vefp. 444.

[k] Hift. liv, ii. 6. 
$244 \quad$ On cn Egyptian Colony eftublifhed at Athens.

"frivulofa elt antiquitas, biformem tradidere, qui primus marem " foeminae matrimonio junxit."

IL y en a enfin qui difent que c'eft à caufe de fa grandeur gigantefque, par laquelle il furpaffoit de la moitié les autres homes; ou peut-être parce qu'il favoit les deux langues, la Greque et l'Egiptienne.

VoIci des vers de Jean Tfetzes à ce fujet[l].

"Primus omnium in Attica regnat Cecrops,

"Primus qui ( $\Delta$ เфù's) biformis dictus fuit, in hunc modum;

"Vel quod magnitudine duorum hominum proceritatem aequaret,

"Vel quod Graecae linguae et Aegyptiae peritus, \&c."

Toutes les explications, que je viens de raporter, peuvent avoir leur fondement et conviennent au mot Sigraph, ou $\Delta$ squ's; il fe peut même, que fuivant la conjecture de Milord Comte de Winchilfea $[m]$, on doit expliquer, fur ce fondement, les doubles têtes qu'on voit fur quelques médailles d'Athènes. Ces têtes adoffées; repréfentations emblèmatiques, paroiffent être d'origine Egip. tienne, d'où elles furent portées en Grèce et en Etrurie; leur explication eft fort obfcure, et je n'ai rien de nouveau à ajouter fur leur fujet, à ce qui en a été dit en dernier lieu par Mr. le Comte de Caylus [ $n]$.

L'ARRIVEE de Cecrops à Athènes eft le commencement de l'Ere Attique, et la principale date des Marbres. de Paros. Ce cher et précieux monument de la chronologie ancienne met $\mathrm{Ce}$ crops 373 ans avant la prife de Trö̈e. Eufebe le met 375. Syncelle, au contraire, 6I6. Mais de quel authorité peut être cet auteur, quand il eft en contradiction avec les marbres d'Arundel, avec Eufebe, et avec Cenforin, qui après l'heureufe reftitution [0]

[l] V. Hift. Chil, ver. 18.

[m] Haym Teforo Britannico, part i. pag. 156.

[n] T'um. II. pag. I49.

[0] Reftitution Chron. d'un endroit de Cenforin par Mr. Boïvin l'ainé dans les Mem. de Lit. de l'Ac. R. des Infc. Tom. IV. pag. 33, et fuivantes. 
qu'en a fait Mr. Boivin, met un peu moins de 400 ans, depuis Cerops jufqu'à la-prife de Troïe. L'arrivée de ce colonifte Egiptien peut donc être placée avec Selden et Marham [ $p]$ dans l'année $3^{1} 3^{2}$ de la periode Julienne.

Honorius, Freculphus, et Auguftin, difent, que le peuple d'Ifräel fortit d'Egipte dans le tems que Cecrops règnoit à Athènes, ce qui convient à l'antiquité que je viens de donner à ce héros.

Cecrops porta aux Grecs les coutumes et les rites Egiptiens; principalement le refpect pour les bêtes animés, dont il ne vouloit pas qu'on fit ufage pour les facrifices. Il défendoit de même l'ufage du vin dans les ofrandes, fuivant les coutumes des Egiptiens, dont les égards pour les animaux, et l'horreur pour le vin font conus. C'eft ainfi que Paufanias $[q]$ nous apprend, que $\mathrm{Ce}$ crops n'a pas voulu qu'on immolat à fon Dieu Jupiter des chofes qui euffent eu la vie; mais feulment des liba, ferta, ou gateaux, que les Atheniens appelloient $\approx \varepsilon \lambda \alpha^{\prime} y \xi^{\prime}$.

MAIs comment concilier ce que j'avance avec Eufebe [r], et plufieurs autres anciens, qui difent, "que Cecrops ofrit des boeufs à " fon dieu[s]?" Le grand Meurfius a parfaitement levé cette contradiction apparente, en faifant voir que ces $\Pi^{\prime} \lambda_{\lambda} \alpha \nu$ or, ou gâteaux, des Athéniens $[t]$, dont on fe fervoit dans les facrifices avoient des cornes, et que par cette raifon on les apelloit des boeufs; qu'on leur donnoit cette figure, par ce qu'on les mettoit et ofroit à la place de ces animaux fi.honorés en Egipte. C'eft de cette façon qu'on peut expliquer ce que Diogene Laërce $[u]$ raconte, que l'Empedocle immola aux dieux un Boeuf, de miel, et de farine.

[ $p$ ] Marhham, § viii. Seld. Com. fur les marbres d'Arundel.

[q] Arcadica.

[r] Ifid. Hifpal. Orig. viii. I I.

[s] Cedrenus, Gotfridus Viterbienfis, Chron. partie iv. de Regg. Athen. ch. 9.

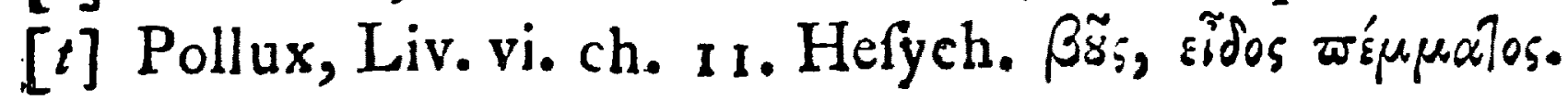

[u] Liv. viii. ch. 53 . 
$246 \quad$ On an Egyptian Colony eftablifised at Athens.

ON ne faifoit point ufage du vin au rapport de Paufanias [w] dans les facrifices de Jupiter, dont le culte fut introduit par $\mathrm{Ce}$ crops. Ce heros eft mis par cet endroit dans le Zodiaque, a la place de l'Aquarius; c'eft ce que Hygin [x] nous aprend, en difant: - Eubulus autem Cecropem Aquarium effe demonftrat, anti." quitatem generis commemorans, et oftendens, antequam vinum " traditum fit hominibus, aqua in facrificiis deorum ufos effe, et " ante Cecropem regnaffe quam vinum inventum fit." Euripide[y] met de même la coupe de l'Aquarius dans les maius de Cecrops.

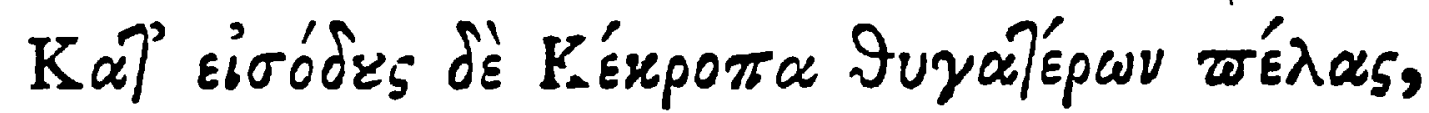

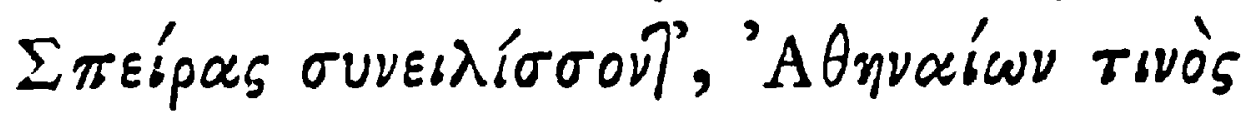

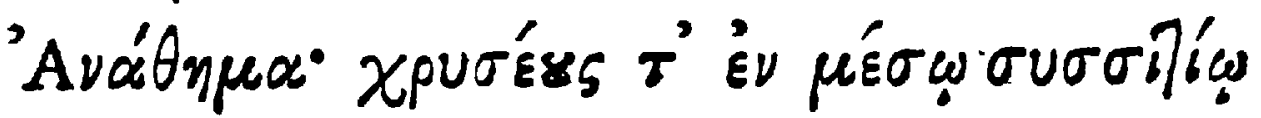

$\mathrm{K} \rho \alpha] \bar{\eta} \rho \alpha \varsigma^{\prime \prime}{ }_{5} \zeta \eta \sigma^{\prime}$.

"Ad januam vero Cecropem prope filias,

"Involutum nodis, Athenienfium cujufdam

"Donarium; aureofque in medio convivio

" Crateres ftatuit."

Plutareue [z] nous apprend, que les Egiptiens avoient le vin en horreur, le regardant come le fang des impies, qui firent autrefois la guerre aux dieux. C'eft de là que vient l'abomination [a] que les Mages, les Gnoftiques, les Arabes, les Brachmanes, et les Moines de la Chine, ont pour le fruit-des vignes; et c'eft pour diftinguer fon peuple du refte des nations idolatres, que Dieu a introduit fous le Vieux Teftament l'ufage du "vin, auffi fréquent dans fes ofrandes.

[w] In Atticis.

[x] Poet. Aftron.

[y] In Ione, ver. 1165 .

[z] De If. Tom. II. pag. 353.

[a] M. Michaelis s'etend fort au long à ce fujet dans les Mem. de l'Acad. R. de Gottingen, Tom. IV. 108. dans une Differt. qui a pour titre, "De Legibus Mofis "Palaettinam populo caram facturis:" 
On an Egyptian Colony efablifhed at Athens.

Sans entrer dans la difcuffion exacte des fciences et des arts, que ce colonifte aprit aux Athéniens [b], je finirai fon article par ce que Tacite nous aprend; que Cecrops donna un alphabet aux Athéniens $[c]$. Ces lettres font plus anciennes que celles de C̆admus [d], et cet alphabet contenoit les vieilles lettres Attiques. C'eft avec ces caractères qu'étoit gravée l'infcription de Mégare, le plus ancien monument de pierre que Paufanias [e] eût vû en Grèce, et qui fut trouvé dans le tombeau de Corebus, et érigé peu de tems aprés la mort de Cecrops.

DE ce premier conducteur des Egiptiens à Athènes, je paffe au fecond, qui eft Erictonius.

LEs hiftoriens $[f]$ et les chronologiftes anciens font mention de deux diférens rois d'Athènes, qui ont porté le même nom, et qui pour cette raifon ont fouvent été confondus. Ils s'appelloient Erechteus ou Erichtonius. Ces déux noms font les mêmes felon les fcholiaftes d'Homère $[g]$; un d'entr'eux a été le quatriéme, et l'autre le fixiéme, des rois d'Athènes.

$\mathbf{C}^{\prime}{ }_{\text {EST }}$ du prémier de ce deux rois que Diodore de Sicile [b] nous apprend, qu'il étoit Egiptien d'origine; et que dans les tems d'une grande famine qui défoloit l'Attique, ce heròs apporta du bled aux Athéniens, à caufe de l'alliance [i] que ceux ci avoient avec les Egiptiens, ceft à dire, à caufe de la colonie établie auparavant par Cecrops.

Pour le recompenfer de fes bienfaits, les Athéniens le reconurent pour leur roi, et donérent à fon frére Butes le facerdoce de

[b] Annales, Liv. xi. ch. I4.

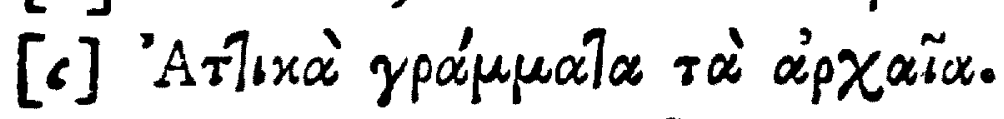

[d] Hefych. Paufan. Liv. iv, ch. 39.

[e] Paufan. Liv. i. ch. 43 .

[f] Sigonius de Tempp. Athen.

[g] Homère, II. B. ver. 552 .

[b] Liv. i. Tom. I. 33. Ed. de Weffeling, et pag. 57. Tom. I. de la Trad. de M. l'Abbé Terraffon.

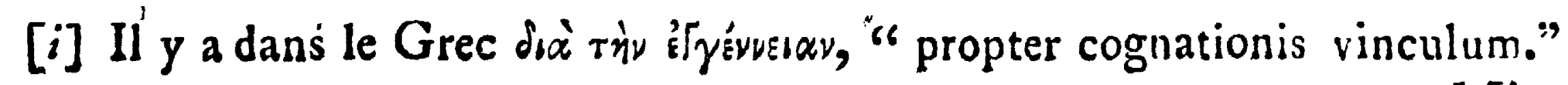

Minerve 
$24^{8} \quad$ On an Egyptian Colony eftabiiged at Athens.

Minerve et de Neptune. On raconte auffí qu'Ericbtonius aprit aux Eleufiniens les miftères de Ceres. Ne pourroit on point expliquer cela du moins en partie de la provifion de bled, que ce colonifte Egiptien leur procura, et de l'art de l'agriculture qu'il perfectiona dans ces pais?

LEs etymologies ridicules des grammariens, qui ont voulu expliquer le nom d'Erichtonius par la langue Grèque, m'ont engagé à chercher plûtôt dans la langue Egiptienne, et dans la patrie de ce heros, l'etymologie de fon nom. Je me flatte, que l'on trouvera affés probable çelle que je vai donner. Le mot ancien Egiptien eft Erichto; de là les Grecs ont fait Erictheus, Ericthonius, come d'Apollo, Apollonius. Ce nom eft compofé de eps facere, rei alicujus auciorem effe, et $200[k]$ chto ou icbto, equus, equitatus. De là Eri-cbto, autbor equitatus et equorum. C'eft l'emploi que les anciens donnoient unanimemént à Erichtonius.

VIrgILE en ces vers, Georg. iii. v. I 3 .

"Primus Ericthonius currus et quatuor aufus

" Jungere equos, rapidifque rotis infiftere victor."

Servius [l], Ariftide, et d'autres confirment tous, que ce heros dompta le premier les chevaux, et les attela à fon char.

C'est pour éternifer la mémoire de cette invention, qu'Ericthonius a été placé après fa mort au ciel, et que dans la fphère des anciens il eft repréfenté fous les noms d'Auriga, d'Agitator, ou de Heniochus. C'eft ce que Germanicus nous aprend dans ces vers, que je cite d’après un manufcrit de la Bibliothèque de Berne, qui

[k] Il y a aparence qu'avant la confone du mot $८ \ominus 0$ cbto, les Egiptiens mettoient la voïelle $i$, ce qui feroit er-ichto.

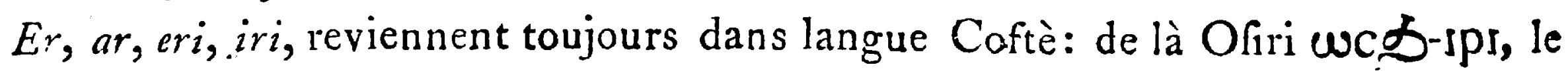
Nil et le Soleil fignifient le dieu, auteur de la moiffon et des fruits. or $\& \oplus 0$

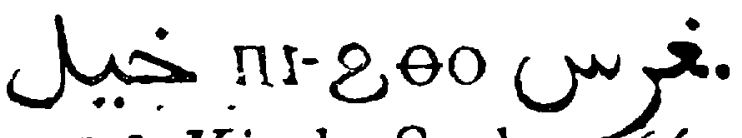

[l] Kirch. Scala, 166. Verfion Cofte, Gen. xl. I7. et x]ix. 19. 
difère ici, come en bien d'autres endroits, de l'edition de $\operatorname{Morel}[m]$, dont jai coutume de me fervir.:

" Eft etiam aurigae facies, five Attide terrâ

“ Natus Erichthonius, qui primus fub juga duxit

"Quadrupedes; feu Myrtoas demerfus in undas,

"Myrtilus hunc potius fpecies in fidere reddit.

"Sic nulli currus, fic ruptis moeftus habenis,

"Perfidia Pelopis raptam gemit Hippodamiam."

Vorcr ce que Hygin [ $n]$ dit à ce fujet: " Heniochus, hunc nos "Aurigam Latine dicimus nomine Erichthonium, ut Eratofthenes " monftrat; quem Jupiter, cum vidiffet primum inter homines "equos quadrigis junxiffe, admiratus eft ingenium hominis ad "folis inventa accefliffe." Avienus dans fes Phénomènes le nomme auffi Aurigator, et lui donne cet emploi dans les vers fuivans:

"Ille impiger autem

"Pulcher Erichthonius currus et quatuor aufus

"Junxit equos."

MaIs ce qui confirme le plus mon etymologie, et.répand une nouvelle lumiére fur cette colonie, c'eft qu'Erichtonius et Erichteus, eft un de prénoms des Neptune [0]. Lycrophon, Tfetzes, Hefychius, et d'autres anciens, difent qu'Erichteus eft Neptune parmi les Athèniens: Plutarque $[p]$ en deux diférens endroits fait mention du facerdoce de Neptune Erichthonius $[q]$. Ce dieu eft

[m] N'aïant point vû l'edition de Germanicus publiée par Grotius, je ne fai fí ces variations s'y trouvent déja.

[n] Poet Aftron. II. 13.

[0] Caffandre, verf. 158. Coment. de Tfetzes, pag. 32. Edit. Greque de Bàle.

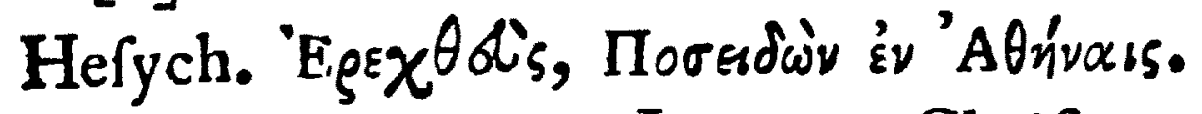

$[p]$ Athenagoras, Leg. pro Chrift.

[q] Plutarque, Vie de Lycurgue.

Vol. I.

$\mathbf{K}$ k

$\operatorname{ain} x$ 
$25^{\circ}$ On an Egyptian Colony eftablifhed at Athens.

ainfi nommé, fuivant $\mathrm{ma}$ cenjecture, parce que c'eft lui que l'on a crû être le premier inventeur et l'auteur de l'equitation des Grecs: Sophocle et Diodore de Sicile atteftent cette verité. Paufanias [r] parle d'une fíatue equeftre de Neptune érigée à Athènes, et pour rendee raifon du prénom i $i \pi s o s$, qu'on donoit anciennement à Neptune, il a recours à l'invention de l'equitation, de laquelle il prouve qu'elle étoit attribuée à ce dieu. Les Hymnes de Homère donerit deux emplois à Neptune, celui de l'equitation, et celui de la navigation.

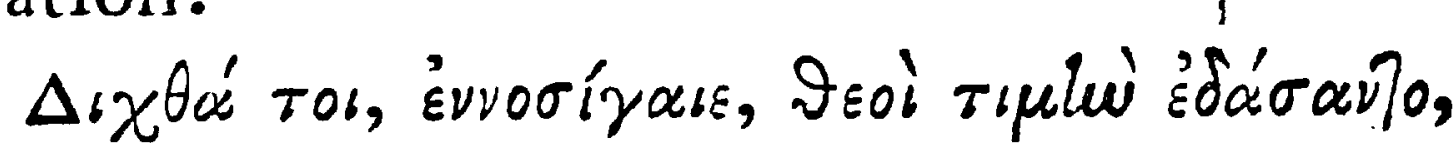

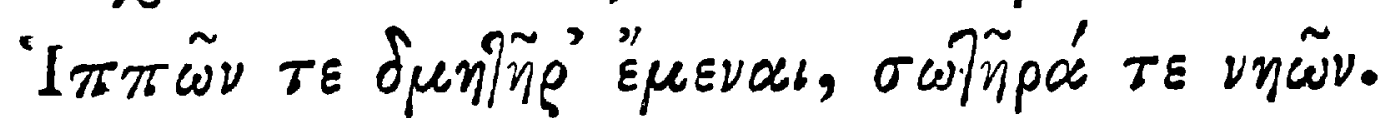

C'E toit la même chofe en Italie: les Etrufques repréfentoient auffi Neptune trainé dans un char; c'eft ainfi que nous le voions dans un monument de cette nation publié par Demfter [s]. C'eft par la même raifon que Romulus dédia à ce dieu les fêtes appellées Confualia [t], dans lefquelles on avoit coutume de couroner les chevaux.

Les anciens vont même plus loin; Neptune n'eft pas feulement l'inventeur de l'equitation, mais c'eft lui qui a crée et produit le premier cheval. Rien de fi comun dans les auteurs de mythologie que la fable touchant Neptune, qui en frappant la terre par fon trident en fit fortir le premier cheval. Deux páffages des anciens poëtes me ferviront ici de preuve. Voici le premier, qui eft tiré de Lucain [u]:

"Primus ab aequorea percuffis cufpide faxis

"Theffalicus fonipes, bellis feralibus omen,

"Exiliit."

VIRGILE $[w]$ nous aprend la même chofe dans le vers fuivans: " Tuque ô, cui prima frementem

"Fudit equum magno tellus percuffa tridenti,

"Neptune."

[r] Paufan. Attica, p. in 2. Achaica, 227.

[s] Tab. 74. et Gorius Muf. Etr. Tom. II. 169.

[t] Denis d'Halicarnaffe, pag. 26. Ed. de Silburg.

[u] Pharf. vi. 396.

[w] Georg. i. 12. 
Ce n'eft donc point fans raifon que Neptune, eft appellé Erechteus. Ce nom lui convient dans toute fa fignification. Il eft non feulement l'inventeur de l'equitation, mais auffi le dieu qui fit préfent aux homes des chevaux; c'eft à jufte titre que Pamphus

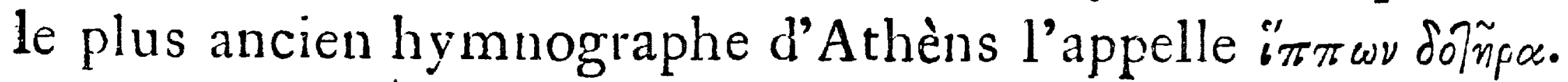

PEu t-être me fera-t'on ici deux queftions; pourquoi, demanderat'on, ce colonifte Egiptien eft il appellé Erechteus ou Neptune? Et pourquoi les anciens ont ils atribué l'equitation à l'un et à l'autre? Seroit-ce parce que les Egiptiens, qui vinrent par mer en Grèce, furent en même tems les premiers auteurs de l'equitation des Grecs? Cette réponfe ne me fatisfait point, et j'en ai une autre qui me paroit plus folide, fondée fur ce; que l'equitation dans les tems les plus anciens étoit l'emblème de la navigation. Cette vérité a été démontrée par Monfieur Fréret, dans fes ingénieufes réflexions fur les fondemens hiftoriques de la Fable de Bellerophon $[x]$ : le Pégafe de ce héros, n'eft, fuivant cet auteur, qu'un vaifleau dont il fe fervit dans fes expéditions. Ainfi Neptune, dans fa difpute avec Minerve, fit fortir le cheval de la terre; c. à d. qu'il conteilla aux Athèniens la navigation. Minerve de fon côté en fit fortir l'olivier; c. à d. qu'elle confeilla l'agriculture.

$D_{E}$ là il réfulte, qu'Erichthonius qui arriva en Grèce avec des vaiffeaux chargés de bled, étoit ainfi nommé, parce que d'Egipte il vint par mer pour foulager les Atheniens fes anciens compatriotes.

QUELLE pourroit être enfin la raifon pourquoi les anciens ont repréfenté la navigation fous l'embléme de l'equitation $[y]$ ? Eft ce à caufe de la viteffe de courfes, et de l'affinité qu'ils trouvoient entre ces deux façons de voï̀ger? Eft ce à caufe des répréfentations 'fur la proue des vaiffeaux ? Pourroit on peutêtre l'expliquer de la tête

[x] Hift. de l'Acad. R. des Infc. et Belles L. Tom. IV. Ed. d'Amft. pag. 57. et fuiv.

[y] Voies la deffus le docte Comment. de Meurfius fur Lycophron pag. $33^{6}$.

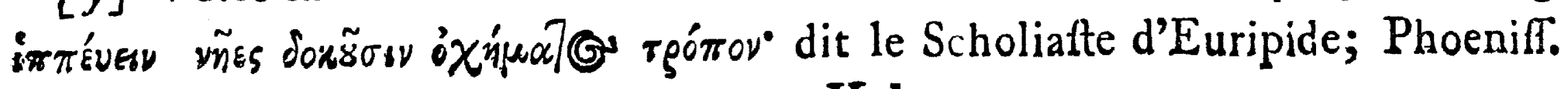

$\mathrm{Kk} 2$ 
52 On an Egyptian Colony. eftablifhed at Athens.

de cheval fimbole de Carthage, ville fondée par u i:e flotte de Phé. niciens? Seroit-ce de cette manière, qu'on doit entendre la prife de Trö̈e atribuée au cheval de bois, c. à d. à un vaiffeau rempli de foldats. Il paroit que Plaute $z j$ fait allufion à cette $f_{d}$ ble en difant:

"Nempe equo ligneo per vias coerule as

" Eftis vecti."

L a mémoire d'Erechteus, et fa gloire, fe fortt confervées jufques à nos jours. Nous admirons et contemplons avec étonement les beaux reftes du magnifique temple [a] érigé à fon honeur à Athènes; monument qui faifoit autrefois, et qui fait encore aujourd'hui un des plus fuperbes ornemens de cette ville.

IL y a encore un troifiéme colonifte, qui eft Petes. Diodore de Sicile [b] nous aprend qu'il étoit Egiptien, Pére de Meneftheus, onziéme roi d'Athènes, qui perdit la vie au fiége de Trö̈e.

Nous favons fort peu de chofe de ce Pétes; et je ne m'arrêterai qu'à fon nom, qui me paroit compofé de $\operatorname{Pet}[c]$, qui fignifie Prêtre, et es, is, Ifis. Pétes au lieu de Petifis, " facerdos Ifidis." Nous connoiffons un Petifis Egiptien, dont Arrien fait mention dans l'expédition d'Aléxandre le Grand; Jamblique [ $d$ ? nous a auffi confervé la mémoire d'un Bytis mifte Egiptien. J'explíque de même les noms propres Petofiris $[e]$, prêtre d'Ofiris; Yetefuchus $[f]$, prêtre du Crocodile; Petencit, prêtre de Minerve; et Petephre,

[z] Rud. Act. I. fc. 5. 1. 10.

[a] On en voit les plans dans les deffeins d'antiq. d'Athènes publiées en Angleterre par Richard Dalton.

[b] Liv. I. pag. 33 .

[c] Les réduplications dans les mots Egiptiens font fouvent omifes. On peut fort bien dire Petes pour Petifis.

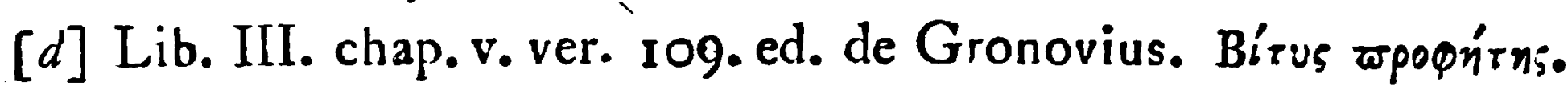

[e] V. Jamb. de Myfter. pag. 161. ed. d'Oxford, par Th. Gale.

[f] Plin. H. N. XXXVI. I3. 
On an Egyptian Colony eftabliged at Athens.

prêtre du Solfil. Le fils de ce Petes eft Maneftheus; ce nom eft encore $E_{\tilde{c}}$ iptien, et le même que celui du famelix Minethos.

$\mathrm{C}_{\mathrm{E}}$ font là les chefs des Athéniens qui vinrent d’Egipte. Diodore de Sicile qui en fiii l'cnumeration omet Cecrops, 'et' apelle Petes $\Delta$ ‘qùns; mais Palmerius, Marham, et Weffelingius [ $g]$, ont déja remarqué, que le texte de cet auteur eft corrompu, et que ce pronom convient à Cecıops, qui a été oublié par les copiffes.

III. Je paffe à la derniére preuve de cette colonie, foncée fur le raport de Platon [b], qui nous aprend que la principale déuffe de Saïs étoit Neitha, que les Grecs lui donnoient le nom d'Ailwãx, et les Latins celui de Minerve. Cecrops et ces coloniftes portérent de Saïs le culte de cette divinité à Athènes [i]. On voïoit, à ce que nous dit Paufanias" $k$ ], un temple érigé en Gréce à l'honeur de la Misierve de Saïs. Cette déefle eft fort ancienne en Egipte: ou l'adorcit déj du tems du patriarche Jofeph. Je le prouve par le nom de fa femme Afenet [l], qui fuvant la conjecture de Mr. Jublonki fignifie "cultrix Minervae." Le nom de cette divimlée étort Veit, Nir, Net, Neitha $[\mathrm{m}]$; de là dérivent les noms propr ss Egiptiens, Nittis [n], Pfammeniti et Pateneit [o]. Il eft difficile de doner un etymologie probable de ce mot; plufieurs favans l'ont tenté, et celle de Mr. Renaudot [p] confirmée, en dernier lieu, par un favant anonyme me paroit la

[g] Coment. fur D. de Sicile, Tom. I 34 .

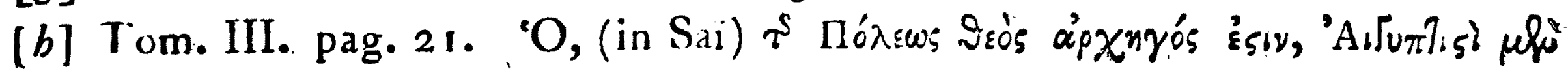

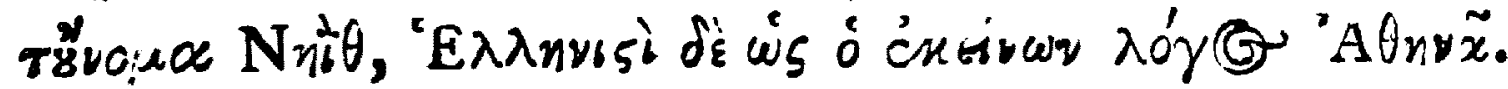

[i] Cicero, Nat. Decr. L. I.I. " Minerva fecunda orta Nilo, quam Aegipti "Saïtae colunt."

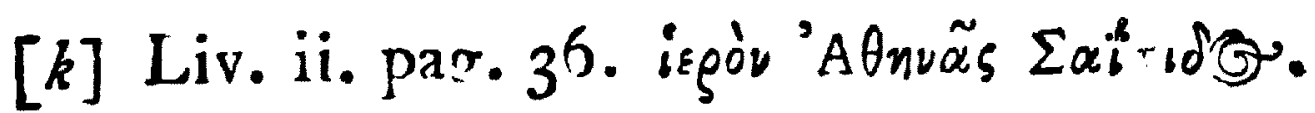

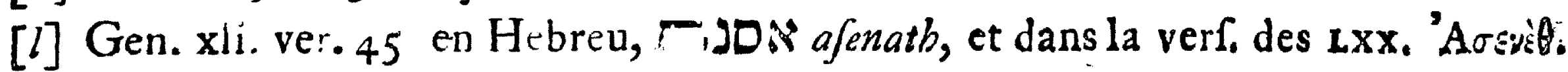

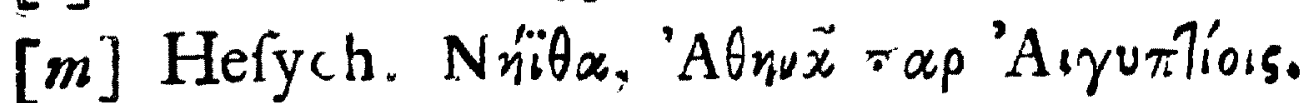

[n] La Crofe Epitt. Tur.. III. pag. 155.

[o] Tabl. Parth Tom. I. pag. 76.

[p] Mem. de l'Acad. des infc. Tom. II. pag. 339. edito d'Amft. 


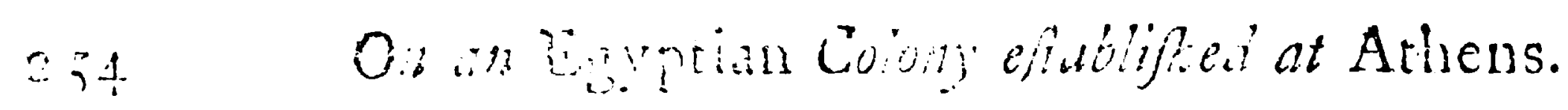

Itus proba'le. Il fi.it dériver ce not de Nauti, Deus, ens fummum [q]. Je n'entre point dans la difcuffion du mot 'A A contentant de remarquer, qu'un auteur moderne croit y voir les veftiges du mot Neitha.

Que la Minerve d'àthènes foit dans fon origine la même que celle des Eziptiens, c'eft ce qui ne peut être nié, après les témoignages que je viens de raporter; mais il ne faut point croire, que tout ce que les Grecs ont atribué à leur Minerve foit venu d'Egipte.

IL y a, par exemple, une grande difpute entre M. Jablonfki [ $r]$ et l'Abbé Pluche [s] fur la queftion fi l'olivier parmi les Egiptiens étoit confacré à Minerve, et fi ceuxci atribuoient à cette déefe l'invention de l'huile d'olives, come ont fait les Athéniens. Ce dernier auteur pofe ce fait pour certain, quoique tous les paffages des anciens auteurs $[t]$, qui méritent toujours nos égards et nos attentions, concurrent à n', us aprendre qu'il n'y avoit point d'oliviers dans la baffe Egipte $[u]$, et que cet arbre étoit confacré en Egipte, non à Mineirve, mais à Mercure.

LA grande preuve de Mr. l'Abbé Pluche et de fes partifans eft le nom de Sä̈s[w], qui en Hebreu et en Egipt en fignifie olivier. Je paffe fous filence quelques autres etymolosies probables, que

[q] Naturae et Script. Concordia, pag. 240. On croit que l'auteur de ce fcavant traité eft Mr. Wachter.

[r] Pantheon, Tom. I.

[s] Hift. du Ciel, I. p. I84.

[t] Strabon, liv. xvii.

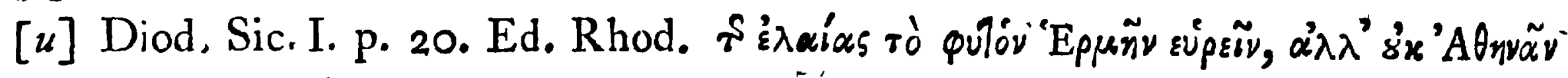

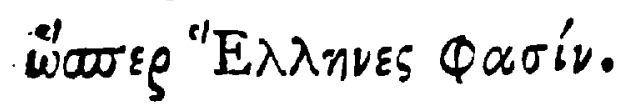

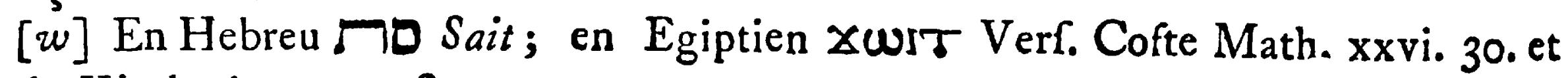
Scala Kircheri, pag. 178.

La prononciation de la lettre $\times$ Giangia en langue Cofte eft difficile, et je crois qu'on ne feroit pas mal de la comparer avec le ice, qui fe trouve dans l'alphabet Arménien parfaitment fous la même forme, dont la prononciation eft par un François $d g$ ante $e$, aux Anglois $j$ confone, aux Allemánds $i f c h$. XWrT, doit donc être prononcé Ifchoit. 
jaurois du mot de Sä̈s, et j'aim: mieux faire une petite digreffion pour refuter cet argument, qui, fuivant les auteurs, ferve d'un cô:é, â établir que l'olivier étoit confacréà Minerve, et enfuite à prouver cette affinité des langues Hebraique et Egiptienne, qui quoique entiérement imaginaire, ne laiffe pas d'être crüe par bien des favans.

Je détruirai ces deux conféquences par le raifonement fuivant, fondé fur ce, que nous favons par le raport des anciens, que les Egiptiens ont eu leur huile d'olives de la terre d'Ifrael $[x]$. Les. Juifs trafiquoient avec cette marchandife en Egipte; ils avoient peut être leur entrepot d'huile à Saìs; cette marchandife étrangéré a confervé fon nom en Egipte; tout come les fruits et les drogues qui nous viennent de paîs étrangers gardent les noms qu'ils poffédoient dans leur païs natal. Ainfi l'huile eft appellée Saït, non feulement dans tous les dialectes qui ont du raport avec l'Hebreu, mais auffi dans les langues des Egiptiens et des Arméniens $[y]$; dont châcune difère entiérement de toutes les langues conües. Les. Egiptiens s'étant enfuite établis à Athètues plantérent des oliviers. qui y réuffirent; ils les dédiérent à Minerve, non point pour fuivre en cela les coutumes Egiptiens, mais fimplement fuivant les ufages. des anciens, de confacrer ce qu'il y a de plus célèbre dans un païs à fa divinité principale et protectrice.

$\mathrm{J} E$ ne puis paffer fous filence une petite obfervation fur le mot xwrt, qui comme je viens de le prouver fignifie l'olivier. Ce mểme mot fe trouve au pluriel dans le Dictionaire Cofte et Arabe du P. Kircher [z], où il eft traduit par " fornicatores, adulteri." J'ai découvert l'origine d'une traduction fi fautive. Kircher a lâu. dans l'Arabe, Alzanion; où il auroit falu lire Alzeitunon. Cette correction ne dépend que du changement de points, la figure des. lettres refte entiérement la même. Voilà qui prouve le peu d'at ..

$[x]$ Kimchius in Hofeae, ch. xii. I.

[y] En Armenien Efeth.

[z] Pag. 440. H1-xwis نالزيون lifez 


\section{$25^{5} \quad$ On an Egyptian Colony eftablifbed at Athens.}

tention qu'a eu Kircher en publiant fon livre, et qui fait voir, en même tems, là nécefifité de la connoiffance de la langue A rabe pour fe ferver utilement de cette ouvrage. Mais venons à notre fujet.

UN $\mathrm{F}$ autre affinité qu'on fuppole entre la Minerve des Egiptiens et celle des Grecs, c'eft qu'on dit que la Minerve des Egiptiens étoit vierge, come celle des Grecs. On le prouve par le témoignage de Plutarque, qui cite une infcription de Saïs, dans laquelle Minerve fe vante que jamais perfone n'a relevé fon voile. Le grand Molheim, et le favant Jablonfki, ne veulent point admettre cette infcription; ils la regardent come forgée d'après la théologie Grècque. Je ne me fervirai donc de cette preuve, qui foufre quelques difficultés; auffi n'en ai je pas befoin, aïant des conformités fúres et certaines, qui prouvent que la Neitha de Saïs eft la même que la Minerve des Grecs [a].

Minerve, déeffe guerriére des Grecs, étoit honorée fous cette même qualité en $E_{\varepsilon}$ ipte: je ne prétens point prouver ce que j'dvance par les medailles de Saïs, où l'on voit Minerve armée d'un calque et d'un lance; les medailles de l'Egipte frappées fous les Empereurs Romains, ne font point les véritables réprélentations des dieux Egiptiens!b]; mais je le prouve par ce que Procle $c]$ nous dit, que la Minerve des Egiptiens et des Grecs ont l'une $t$ t l'autre les deux qualités d'être guerriéres et philotophes à la fois. Je le prouve auffi par ce que nous favons, fuivant le témo:gnage d'Ho. rapollo $[d]$, que l'efcarbot étoit l'emblême de cette déefle. Or l'efcarbot, à ce que nous aprennent Plutarque $[e]$ et Elien [ $f]$, étoit 1 - fimbole des guerriers, et lervoit ordinairement de cachet aux foldats Egiptiens.

[a] Coment. fur le Siftème de Cudwort, 398.

[b] Vaillant Aeg. numifm. pag. 2I4.

[c] Coment. fur le Timé, pag. 30.

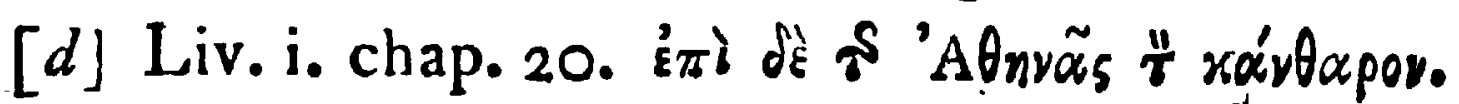

[e] De If. et Or. 355 .

[f] Hif. An. X. I5.

Dans 
Dans le catalogue d'Eratofenes[e], confervé par Manetbon, nous voions une reine d'Egipte nommée Nítwrǫs. Ce mot fig-

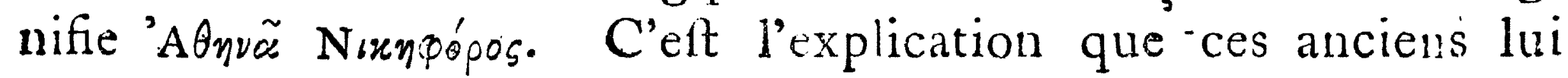
donne, et qui trouve fa confirmation dans la langue Cofte. Qu'on compare à préfent avec ce ittre les médailles Athèniens de Milord

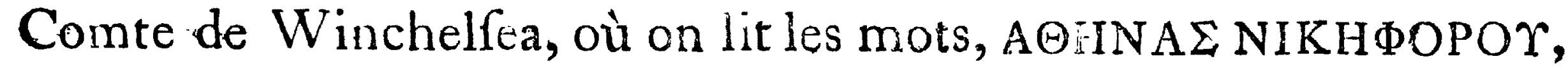
"Minervae viatricis $[f] . "$

Mais fuivant le paffage de Procle que je viens de citer, Minerve n'étoit pas feulement ane déefle guerriére, c'étoit aufî̀ une philofophe. Elle ètoit la déeife de la fageffe en Igipte auffi bien qu'en Grèce; foit qu'on veulle dire, qu'ille eft l'efprit qui gouverne l'univers, foit fimplement parce qu'on fait, que la Minerve d'Egipte étoit déeffe des fciences et des arts. Un des arts les plus utiles eft celui de filer et de faire la toile; c'eft aux Egiptiens que nous en fommes redevables; et c'eft à leur Minerve que ce peuple, comme les Grecs, atribuoit cette belle invention.

Persone n'ignore les beaux habits que faifoient les anciens Egiptiens de leur Byffus $[g]$, dont s'habilloient non feulement leurs prêtres idolatres, mais qui fervoient auffi à orner ceux du vrai Dieu, dont les habits étoient de Schefch Egiptien; car ce mot, fi vous ajoutés l'article $\mathrm{Bi}$, eft entiérement le même que celui dề Biffus.

La coutume de porter ces habits de lin, coutume originaire d'Egipte, s'eft confervée, fuivant Thucidide, jufques au tems de la premiére guerre du Péloponèfe.

Eusta the, dans fes commentaires fur l'Iliade d'Homère [ $b$ ], nous aprend, qu'une femme Egiptienne fit la premiére [i], des ouvrages de tifferanderie, et qu'elle travailla affife à fon métier; il ajoute

[ej Vignnole, Chron: Tom. II. pag. 755.

[f] Haim Teforo Brit. Tom. II. pag. 78.

[g] Maimonides in Hilch Kele Hami. ch. viii. "ubicunque in lege occurrit ש שchefch aut bad, intelligitur Linum et quidem Byffus.

[b] Il A. pag. 31. edition de Rome.

[i] Thucyd. -1. i.

V.oI.. I. 


\section{On an Egyptian Colony eftablifhed at Athens.}

que c'eft ainfi que les Egiptiens repréfentent leur Minerve. Nous le voions en éffet dans la table Ifraque $[k]$. C'étoit auffi la même chofe en Grêce, où, fuivant Strabon [l], les ftatües de cette déeffe étoient toutes affifes. On en voïoit de pareilles à Chios, dans la Phocide, et à Marfeille, et on en voit encore aujourd'hui de femblables en Italie $[\mathrm{m}]$. C'étoit là le plus grand degré de perfection. Il paroit que les tifferans poftérieurs avoient oublié cette maniére de travailler affis; puis que dans les monumens anciens qui nous repréfentent des tifferans, comme font le Virgile du Vatican, et les images peintes d'un Comentaire de Job de la bibliothèque du Roi, nous voions toujours le tifferan debout, tenant dans $f_{a}$ main, non une navette, mais feulement un petit bâton, qui peut être a donné l'origine à ce bâton qu'on voit fouvent dans les mains de Minerve, et que les antiquaires prennent ordinairement pour un bâton de commandement.

Monsieur $1^{2} \mathrm{~A} b b e ́$ Fontenu [n], dans une piéce de goût, publiée dans les Mémoires de Litterature de Paris, me fournit une nouvelle preuve de l'affinité de la Minerve des Egiptiens'avec celle des Grecs; en ce que les Athéniens faifoient voir à la fête des grands Panathenées [o], un vaiffeau, fur lequel étoit l'habit miftérieux de la déeffe. Seroit ce pour marquer la navette des tifferans? Cette opinion feroit probable à caufe du voile de Mínerve, qui étoit pofé fur ce vaiffeau; mais il eft beaucoup plus vraifemblable, que c'étoit ou pour marquer que le culte de Minerve étoit étranger à Athènes, venu au de là de la mer; ou plûtôt à l'imitation de ce fameux navire d'Ifis, fi célèbre en Egipte; car l'Ifis Egiptienne eft, au rapport de Plutarque[ $p]$, la même que la leur; et la Minerve

[k] Littera Z. edition de Frifius.

[l] Liv. xvii.

[m] Gallerie Juftinienne.

$[n]$ Tom. Vil. fur l'Ifis adorée chés les Suèves.

[0] Meurfius Panathenea, dans le Trefor de Gronovius, Tom. II. p. 97.

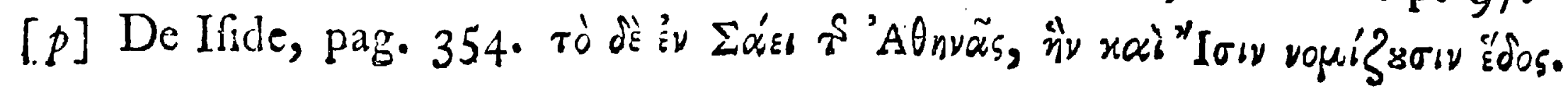


des Grecs eft anffi Ifis, au raport d'Ulpien; le vaiffeau d'Ifis appartient à cette déeffe, parce qu'elle eft la lune [q]; car on fait, que les Egiptiens repréfentoient les aftres dans des navires.

Dans ces mêmes fêtes des grands Panathées je trouve encore d'autres raports avec les pompes Ifiaques des Egiptiens. Les filles, qui portoient de l'eau dans des cruches $[r]$, appellées ígeraópol, étoient en imitation de ces Egiptiennes, qui, dans les fêtes d'Ifis, portoient dans des vafes l'eau du Nil, le cher epoux de cette déeffe.

Les Egiptiens prétendoient, fuivant Diodore de Sicile [s], qu'Ifis avoit inventé plufieurs remèdes fouverains, et qu'elle avoit une parfaite connoifiance de la medicine $[t]$; ils ajoutoient même depuis, qu'elle jouiffoit de l'immortalité $\left[u_{j}^{\dagger}\right.$; elle prenoit plaifir à paroitre, pendant le fomeil, aux hommes, qui imploroient fon fecours dans leurs maladies. Voila donc encore une nouvelle preuve, qui confirme que la Minerve des Grecs eft la même qu'Ifis [w], ou Naitha des Egiptiens. Minerve avoit, en qualité de déeffe de la médecine, des temples en Grèce $[x]$; elle en avoit de même à Rome $[y]$, par le même endroit; et 'c'eft pour cette raifon, que dans les monumens $[z]$ des anciens, nous la voions fouvent accompagnée des ferpens. Je prévois qu'on me demandera, fi les difputes de Minerve avec Neptune font originaires d'Egipte, ou fi c'eft une invention Grècque? Ou je me trompe fort, ou ces difputes ne font

[q] Ulpien fur Démofthène contra Midiam.

[r] Meurfius Panath. pag. I02.

[s] Tom. I. liv. i pag. 29.

[t] Gruter Thef. pag. 83. 15. Minerve eft nommée SALVTARIS. Les Grecs l'appellent ' $\Upsilon$ yissa.

[u] Voiez le Coment. de Broekhoufe fur Tibule, 1. iii. pag. 27.

[w] Paufanias.

$[x]$ Montfauc. Diar. Ital. pag. I 21.

[y] Rufus et Victor in Reg. Efquilina.

[z] La Chauffe, Pierres gravées. 
$260 \quad$ On an Egyptian Colony eftablijed at Athens.

que des reftes des traditions Egiptiennes, touchant la guerre d'Ifis et Ofiris fon epoux, avec Typhon, qui dans leur fiftème étoit principalement la mer: ce que les témoignages de Plutarque mettent hors de doute $[a]$. C'eft de là, que ce peuple avoit en horreur les mariniers, le poiffon, le fel de la mer, qu'ils appelloient Ecume de 'Typbon. C'eft auffi par la même raifon que ce géant étoit crû de couleur rouffe, qui eft celle de la mer voifine du païs.

$\mathrm{O}_{\mathrm{N}}$ m'objectera, peut être, que j'ai tort de confondre le dieu Neptune avec un monftre tel que Typhon; mais Hérodote [b] répondra pour moi, en difant, que les Egiptiens n'ont point connu le dieu Neptune, c. à d. que la mer n'étoit pas une divinité adorée en Egipte; mais plûtôt qu'elle y étoit le monftre le plus détefté. de la nature. Virgile fera garant de ce que j'avance, quand, dans fon Ciris $[c]$, il attribüe à Minerve la victoire fur Typhon.

" Ergo Palladiae texuntur in ordine pugnae:

"Magna giganteis ornantur pepla tropaeis:

" Horrida fanguineo pinguntur proelia cocco::

" Additur aurata dejectus cufpide Typho."

IL y a eu des favans [d] qui ont avancé que le nom de Neptune vient de Neptin, divinité maritime des Egiptiens; et femme de Typhon. Sans difcuter la probabilité de cette etymologie, ’’jajouterai feulement, que fuivant ma conjecture, cette même victoire que je viens de décrire, eft repréfentée fur un monument Etrufque, publié par Monf. le Comte de Caylus $[e]$, avec cette feule différence, que la déeffe pourfuit, non Neptune ou Typhon, mais Hermanubis fils illégitime de Neptin.

JE finirai cette differtation par l'ingenieufe conjecture d'Olearius $[f]$, qui faifant fond fur cette colonie de Saites à Athènes,

[a] Piutarque de Ir, et Or. pag. $3^{6} 3$.

[b] Livre ii. chap. 50.

[c] V. 29 .

[d] Cudworth's True Intellectual Syftem, pag. $3^{10 .}$

[e] Rec. d'Ant. Tom. II. Pl. xx. No 3 .

$[f]$ Godfrid. Olearii Diff. de geftis Pauli ap. Athen. parag. 19. 
tâche de répandre une nouvelle lumiére fur le dieu inconnu, dont il eft fait mention aux Actes des Apôtres [ $g]$. Il croit que ce dieu eft la Neitha des Egiptiens, divinité qui repréfentoit l'entendement invifible et éternel. Cet auteur tire fa grande preuve des infcriptions de Sais, qui fe trouvent dans Plutarque et Procle [b]: dans celle de ce dernier auteur la déeffe parle ainfi: $\tau \alpha^{\prime}$ o

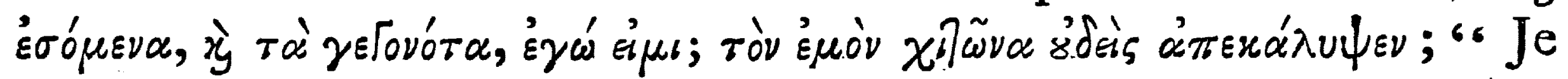
" fuis ce qui-eft, ce qui fera, et ce qui a été; perfone n'a relevé " monhabit." Voilà, dit Olearius, le dieu inconu; et on doit encore remarquer que Jefus Chrift, au quel St. Paul dit que ce nom

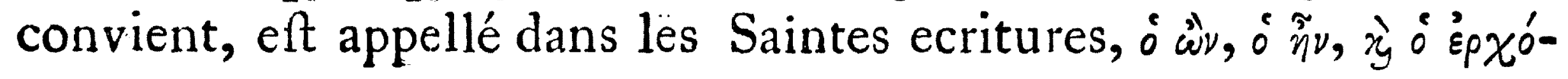
$\mu \varepsilon v{ }^{\prime}$, " celui qui eft, qui a été, et qui fera ;" et que c'eft dans ce

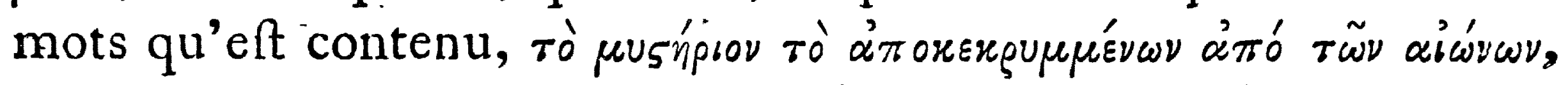
" le miftère couvert d'un voile épais que perfonne n'a relevé.".

$L_{A}$ Neitha des Egiptiens eft donc la même que la Minerve des Grecs. Les coloniftes de Sais portérent fon culte à Athènes; on y voit encore bien des reftes de fon origine Egiptienne; mais on s'àpperçoit auffi des changemens et des additions que les Grecs ont fait, foit par fuperftition, ou plûtôt par ambition, et par orgueil, pour cacher autant qu'il étoit poffible leur véritable origine, qui loin de leur fair tort, leur auroit fait honeur, s'ils n'euffent mieux aimés fe dire nés du fein de la terre, que de reconnoitre ce qu'ils devoient aux Egiptiens leurs ancêtres, dont ils ont imité et furpaffé fi glorieufement les traces, dans l'exercife des arts et la culo ture des fiences.

[g] Chap. xvii. ver. 23

L. Ogmius 